\title{
Strain-dependent variations in spatial learning and in hippocampal synaptic plasticity in the dentate gyrus of freely behaving rats
}

\author{
Denise Manahan-Vaughan ${ }^{1 *}$ and Herbert Schwegler ${ }^{2}$ \\ Medical Faculty, Department of Neurophysiology, Ruhr University Bochum, Bochum, Germany \\ 2 Institute for Anatomy, Otto-von-Guericke University, Magdeburg, Germany
}

\section{Edited by:}

Riccardo Brambilla, San Raffaele

Scientific Institute and University, Italy

Reviewed by:

Martine Ammassari-Teule, Consiglio

Nazionale delle Ricerche, Italy

Kerrie L. Thomas, Cardiff University, UK

\section{*Correspondence:}

Denise Manahan-Vaughan, Medical

Faculty, Department of

Neurophysiology, Ruhr University

Bochum, Universitaetsstr. 150,

MA 4 /149, 44780 Bochum, Germany.

e-mail:denise.manahan-vaughan@rub. de
Hippocampal synaptic plasticity is believed to comprise the cellular basis for spatial learning. Strain-dependent differences in synaptic plasticity in the CA1 region have been reported. However, it is not known whether these differences extend to other synapses within the trisynaptic circuit, although there is evidence for morphological variations within that path. We investigated whetherWistar and Hooded Lister $(\mathrm{HL})$ rat strains express differences in synaptic plasticity in the dentate gyrus in vivo. We also explored whether they exhibit differences in the ability to engage in spatial learning in an eight-arm radial maze. Basal synaptic transmission was stable over a 24-h period in both rat strains, and the input-output relationship of both strains was not significantly different. Paired-pulse analysis revealed significantly less paired-pulse facilitation in the HL strain when pulses were given 40-100 ms apart. Low frequency stimulation at $1 \mathrm{~Hz}$ evoked long-term depression ( $>24 \mathrm{~h}$ ) in Wistar and short-term depression $(<2 \mathrm{~h})$ in $\mathrm{HL}$ rats; $200 \mathrm{~Hz}$ stimulation induced long-term potentiation ( $>24 \mathrm{~h}$ ) in Wistar, and a transient, significantly smaller potentiation $(<1 \mathrm{~h})$ in $\mathrm{HL}$ rats, suggesting that $\mathrm{HL}$ rats have higher thresholds for expression of persistent synaptic plasticity. Training for 10 days in an eight-arm radial maze revealed that $\mathrm{HL}$ rats master the working memory task faster than Wistar rats, although both strains show an equivalent performance by the end of the trial period. HL rats also perform more efficiently in a double working and reference memory task. On the other hand, Wistar rats show better reference memory performance on the final (8-10) days of training. Wistar rats were less active and more anxious than $\mathrm{HL}$ rats. These data suggest that strain-dependent variations in hippocampal synaptic plasticity occur in different hippocampal synapses. A clear correlation with differences in spatial learning is not evident however.

Keywords: long-term depression, hippocampus, in vivo, synaptic plasticity, Wistar, hooded Lister

\section{INTRODUCTION}

Long-term potentiation (LTP) and long-term depression (LTD) comprise forms of synaptic plasticity that occur in the hippocampus in vivo. Both are input-specific, associative, and protein synthesis-dependent (Frey et al., 1988, 1996; Otani et al., 1989; Bliss and Collingridge, 1993; Nguyen et al., 1994; Bear and Abraham, 1996; Manahan-Vaughan et al., 2000) and thus fulfill the criteria for cellular mechanisms for information storage. Both are associated with the acquisition of different aspects of a spatial representation of the environment (Manahan-Vaughan and Braunewell, 1999; Nakao et al., 2002; Straube et al., 2003; Kemp and Manahan-Vaughan, 2004, 2007, 2008; Etkin et al., 2006).

Although rodents readily express different forms of hippocampal synaptic plasticity, differences in the ability of various strains to respond with persistent LTP or LTD following afferent stimulation of Schaffer collateral afferents to the CA1 region have been reported (Manahan-Vaughan and Braunewell, 1999, 2005; ManahanVaughan, 2000a). It is not clear if similar differences exist at other hippocampal synapses. Given the fact that strain-dependent deficits in spatial leaning are associated with strain-dependent deficits in CA1 plasticity (Manahan-Vaughan and Braunewell, 2005) it is of interest to clarify to what extent strain-dependent differences in synaptic plasticity extend to other synapses within the trisynaptic circuit. The dentate gyrus is of particular interest, as it functions as the first stage of the trisynaptic network of the hippocampus, and is believed to engage in pattern separation (Hunsaker and Kesner, 2008). Recent studies showed that the dentate gyrus and CA1 region do not serve identical roles in information processing: different spatial learning tasks are required to facilitate LTD in the dentate gyrus and CA1 regions (Kemp and Manahan-Vaughan, 2008). These regions also do not exhibit identical modifications of synaptic plasticity in response to environment enrichment (Eckert et al., 2010). Furthermore, LTP in the dentate gyrus can persist under certain conditions for many months in adult rats (Abraham, 2003). Despite these intriguing reports as to differences in synaptic plasticity in the dentate gyrus (in comparison to the much better studied CA1 region) little is known about the role of synaptic plasticity in the dentate gyrus, in hippocampus-dependent learning phenomena.

The aim of this study was therefore to examine whether synaptic plasticity in the dentate gyrus reflects performance in hippocampus-dependent spatial learning. To this end, synaptic plasticity was 
evaluated in the dentate gyrus of Hooded Lister (HL) and Wistar rat strains. Spatial learning performance was investigated in the eight-arm radial maze.

\section{MATERIALS AND METHODS}

The present study was carried out in accordance with the European Communities Council Directive of 24 November 1986 (86/609/EEC) for care of laboratory animals and after approval of the local ethic committee (Bezirksamt Arnsberg or Bezirksamt Berlin). All efforts were made to reduce the number of animals used.

\section{ELECTRODE IMPLANTATION}

Seven- to 8-week-old male Wistar and HL rats underwent electrode implantation into the dentate gyrus as described previously (Manahan-Vaughan et al., 1998; Kulla and ManahanVaughan, 2000). The animals were allowed between 7 and 10 days to recover from surgery before experiments were commenced. Experiments were carried out using 9- to 13-week-old rats. Throughout experiments the animals could move freely. Experiments were consistently conducted at the same time of day (commencing $9.00 \mathrm{am}$ ). Baseline experiments to confirm stability of evoked responses were routinely carried out (at least $24 \mathrm{~h}$ ) before LTP or LTD experiments were conducted. Where possible, the animals served as their own controls. Thus, basal synaptic transmission was monitored over a 24 -h period in all animals to confirm stability of evoked responses. Subsequently (i.e., at least $24 \mathrm{~h}$ later), LTP or LTD induction was carried out in the same animals.

\section{MEASUREMENT OF EVOKED POTENTIALS}

Responses were evoked by stimulating at low frequency $(0.025 \mathrm{~Hz}$, $0.2 \mathrm{~ms}$ stimulus duration, $10,000 \mathrm{~Hz}$ sample rate). For each timepoint, five evoked responses were averaged. Both field excitatory post-synaptic potential (fEPSP) slope and population spike (PS) amplitude were monitored. The amplitude of PS was measured from the peak of the first positive deflection of the evoked potential to the peak of the following negative potential. fEPSP slope was measured as the maximal slope through the five steepest points obtained on the first positive deflection of the potential. By means of input/output curve determination the maximum PS amplitude was found for each individual animal, and all potentials employed as baseline criteria were evoked at a stimulus intensity which produced $40 \%$ of this maximum. The input/output curve was obtained by stimulating in a range of 100 through $900 \mu \mathrm{A}$ in steps of $100 \mu \mathrm{A}$.

Long-term potentiation was induced by high frequency stimulation (HFT) at $200 \mathrm{~Hz}$ (10 bursts of 15 stimuli, $0.2 \mathrm{~ms}$ stimulus duration, $10 \mathrm{~s}$ interburst interval). LTD was generated using low frequency stimulation (LFS) at $1 \mathrm{~Hz}$ (900 pulses). The stimulus amplitude for both protocols was the same as that used for recordings.

The baseline fEPSP or PS data were obtained by averaging the response to stimulating the perforant path, to obtain five sweeps at $0.025 \mathrm{~Hz}$, every 5 or $15 \mathrm{~min}$ as described above. The data were then expressed as mean percentage pre-injection baseline reading \pm SEM. Statistical significance was estimated using two-way analysis of variance (ANOVA) with repeated measures. To assess the trial stage at which difference emerged, post hoc Student's $t$-tests were applied. The probability level interpreted as statistically significant was $p<0.05$.

\section{PAIRED-PULSE ANALYSIS}

Before doing eliciting paired-pulse responses, the maximum PS amplitude was determined for each animal by means of the input/ output curve. All potentials were then evoked with the lowest stimulation intensity that yielded a maximum PS amplitude. Five single test-pulse stimuli were given at a frequency of $0.025 \mathrm{~Hz}$ and a pulse duration of $0.2 \mathrm{~ms}$, to confirm the stability of the evoked responses. Paired-pulse responses were obtained by giving pairs of stimuli at interpulse intervals (IPIs) of 20, 25, 40, 50, 100, 300, 500 , and $1000 \mathrm{~ms}$, in ascending order with an interpair interval of $40 \mathrm{~s}$. Paired-pulse ratios were measured between the second PS amplitude and the first one. A ratio of 1 reflects no change in the PS amplitudes evoked by both stimuli. A ratio of greater than 1 reflects paired-pulse facilitation and a ratio of less than 1 reflects depression. A triphasic progression of responses elicits by this stimulation protocol is expected. This reflects an initial paired-pulse depression with short ISIs (20-40 ms), an adjacent paired-pulse facilitation with intermediate ISIs (40-300 ms) and a terminal paired-pulse depression with ISIs of 500-1000 ms. Statistical significance was estimated using a one-way ANOVA. The probability level interpreted as statistically significant was $p<0.05$.

\section{BEHAVIORAL EXPERIMENTS The radial maze}

Performance in an eight-arm radial maze was assessed to determine whether differences in learning performances could be detected in the rat strains.

The radial maze consisted of a central octagonal platform (26 cm in diameter) from which eight arms $(67 \mathrm{~cm}$ long, $20 \mathrm{~cm}$ high, $10 \mathrm{~cm}$ wide) radiated. The floor of the maze was made of dark gray polyvinylchloride, whereas the walls were made of transparent plexiglass. The maze was elevated $80 \mathrm{~cm}$ above floor-level. The end of each arm, possessed a small circular indentation ( $1 \mathrm{~cm}$ deep, $3 \mathrm{~cm}$ diameter) approximately $3.5 \mathrm{~cm}$ from the tip. In the center of this indentation was a $3-\mathrm{mm}$ deep "food cup" ( $3 \mathrm{~mm}$ diameter) in which a $45-\mathrm{mg}$ food pellet could be placed. The indentation served to prevent visibility of the food pellet from the center of the maze. The sides but not the ends of the arms were walled. The walls of the room were 1-2 $\mathrm{m}$ away from the maze, white and had in addition to three cue cards, multiple visual features, such as a door, covered windows, and shelving.

\section{EXPERIMENTAL PROCEDURE}

Nine- to 13-week-old male Wistar rats were used for the behavioral study. For 2 days before commencement of radial maze training animals were habituated to the maze. Food pellets were placed at the end of each radial maze arm. Animals were taken individually from their home cages and placed in the center of the maze 
for $15 \mathrm{~min}$. During these habituation days home cage food access was reduced so that animal weight decreased to $10-15 \%$ of it prehabituation levels.

On training days four arms were baited with a single food pellet ("Dustless Precision Pellet," Bioserv, NJ, USA). For each animal a different constellation of baited arms was randomly chosen. This constellation remained constant throughout the 10-days of training. The trial commenced with placement of the animal on the central platform. A trial was deemed finished as soon as the food pellets had been found or when 15 min had elapsed: whichever occurred first. The number of arm entries was recorded until the trial was finished. The exact position of the entered arms was noted, together with the time spent in the maze, the frequency of freezing and number of rearings. At the end of each trial the number of fecal boli was counted and the maze was cleaned. In order to prevent the use of intramaze cues (such as scent trails) the maze was rotated by $45^{\circ}$ after each training day.

\section{PERFORMANCE SCORING}

Entry into an unbaited arm or entry into a baited arm without removing the food pellet was scored as a reference memory error. Re-entry into a baited arm from which the food pellet had already been retrieved was scored as a working memory error. Re-entry into an unbaited arm was scored as a "double" working and reference memory error (Mizumori et al, 1987; Crusio et al., 1993 ).

\section{DATA ANALYSIS}

With the exception of the first trial day, working, reference, and "double" working and reference memory error data from blocks of three trials were combined for each animal (Prior et al., 1997). The data from the first trial day were presented separately, as this day reflects the first exposure of the animals to the maze task - whereby the animals must learn what the task is before learning can start. The data were then expressed as mean number of errors \pm SEM. The combination of trial days was conducted to obtain more stable results as compared to a single trial day (Ossenkopp et al., 1987; Crusio et al., 1993). The number of fecal boli and grooms per animal were also assigned to trial blocks. Statistical significance was estimated using ANOVA with repeated measures. Within factor analysis was conducted of strain $\mathrm{x}$ trial block. Differences between specific trial blocks was evaluated by Student's $t$-tests. The probability level interpreted as statistically significant was $p<0.05$.

\section{RESULTS}

\section{PAIRED-PULSE FACILITATION BUT NOT INPUT-OUTPUT CURVES ARE} SIGNIFICANTLY DIFFERENT IN WISTAR AND HOODED LISTER RATS

Input-output (I/O) responses were obtained by assessing the amplitude of the PS to stimulation intensities of 100 through $900 \mu \mathrm{A}$. $\mathrm{I} / \mathrm{O}$ curves were not significantly different between strains (each $n=8$;Figure 1A).

Paired-pulse responses were assessed by giving pairs of stimuli at IPIs of 20, 25, 40, 50, 100, 300, 500, and 1000 ms. Responses in HL animals $(n=8)$ were significantly lower in the 40 - to 100 -ms range compared to Wistar rats $(n=8)$, suggesting that paired-pulse facilitation is less in the HL rat strain (Figure 1B, ANOVA, $p<0.05)$.

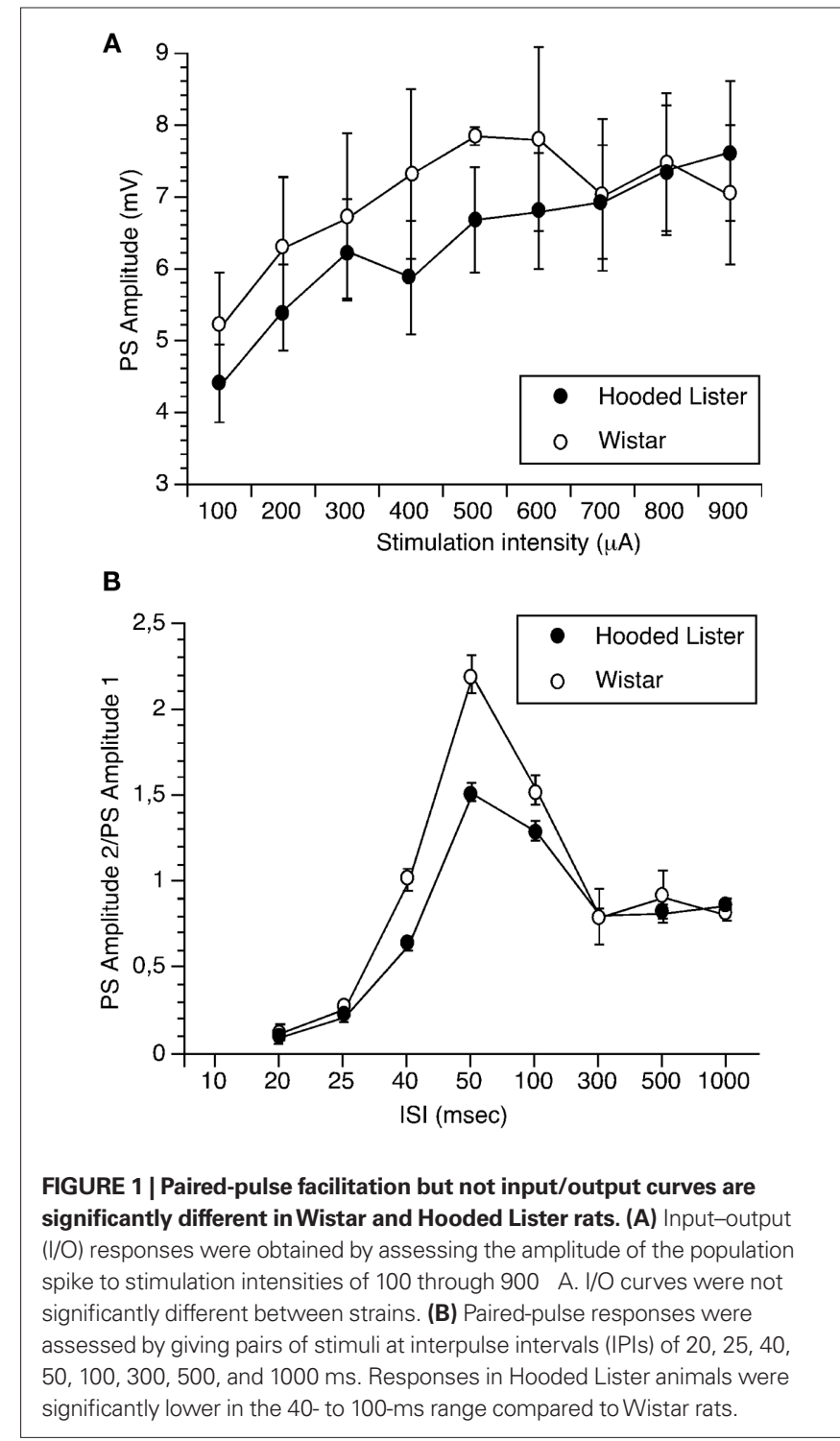

\section{STABLE BASAL SYNAPTIC TRANSMISSION IS ELICITED FOR OVER $24 \mathrm{H}$ IN WISTAR AND HOODED LISTER RATS}

Test-pulse stimulation $(0.025 \mathrm{~Hz})$ elicited stable fEPSP in the dentate gyrus which could be followed for over $24 \mathrm{~h}$ in Wistar (Figure 2A, $n=14$ ) and HL rats (Figure 2B, $n=11$ ). No difference in the profile of responses was observed between the rat strains.

Analysis of variance confirmed a lack of statistical significance between basal synaptic transmission in the rat strains. [For PS, within factor: $F(1,28)=3.549, p=0.1327$; between factor: $F(1,28)=1.286$, $p=0.1788$; For fEPSP, within factor: $F(1,28)=0.801, p=0.421$; between factor: $F(1,28)=1.088, p=0.3663]$.

\section{HIGH FREQUENCY STIMULATION INDUCES ROBUST LTP IN WISTAR BUT NOT HOODED LISTER RATS}

High frequency stimulation (HFS) produced robust LTP in the dentate gyrus of Wistar rats which persisted for over $24 \mathrm{~h}$ (Figures $3 \mathrm{~A}-\mathrm{C}$ ). The same HFS protocol produced only a transient 

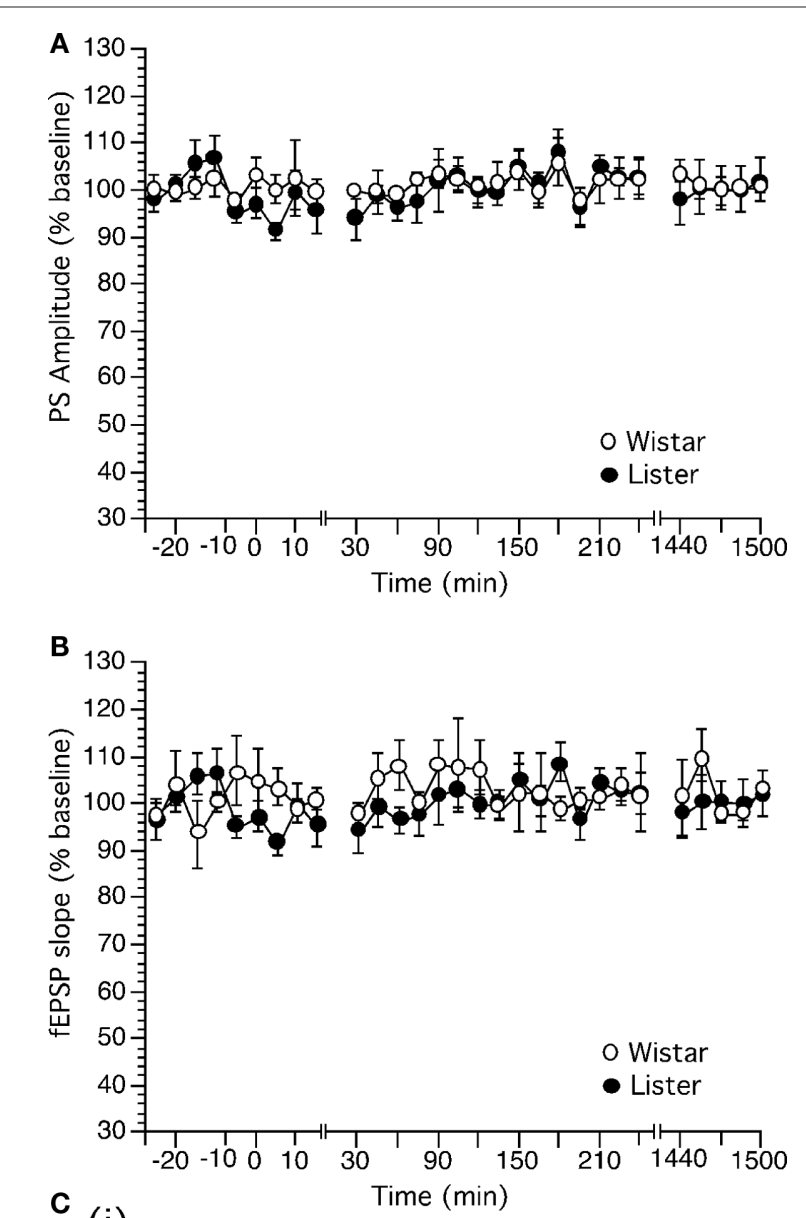

(i)

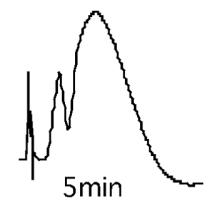

(ii)
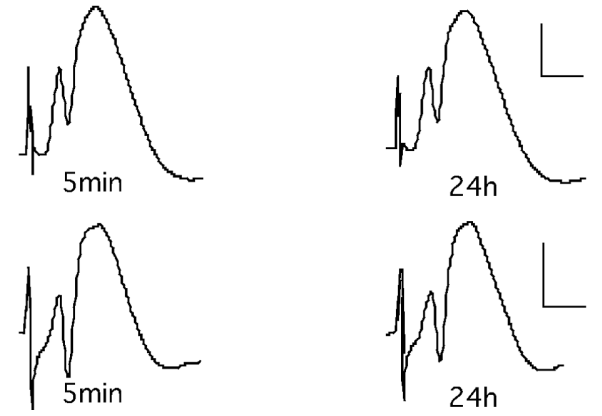

FIGURE 2 | Basal synaptic transmission in the dentate gyrus in vivo can be followed for over $\mathbf{2 4} \mathrm{h}$ in Wistar and Hooded Lister rats. (A,B) Stable basal synaptic transmission was evoked via test-pulse stimulation $(0.025 \mathrm{~Hz})$ in (A) Wistar and (B) HL rats. (C) Original analog traces showing the field potentials evoked from the dentate gyrus following approximately $5 \mathrm{~min}$ and $24 \mathrm{~h}$ following commencement of recordings in (i) Wistar or (ii) $\mathrm{HL}$ rats. Vertical scale-bar corresponds to $5 \mathrm{mV}$, horizontal scale-bar to $4 \mathrm{~ms}$.

potentiation in the dentate gyrus of $\mathrm{HL}$ rats (Figures $3 \mathrm{~A}-\mathrm{C}$ ). This short-term potentiation endured for approximately $90 \mathrm{~min}$ whereupon evoked potentials returned to pre-HFS levels.

Long-term potentiation in Wistar rats was significantly different from baseline controls (each $n=14$ ). [ANOVA, for PS within factor: $F(1,28)=10.155, p=0.0129$; between factor: $F(1,28)=7.774$, $p=0.0001$; for fEPSP, within factor: $F(1,28)=17.954, p=0.0133$; between factor: $F(1,28)=6.178, p=0.0001]$.
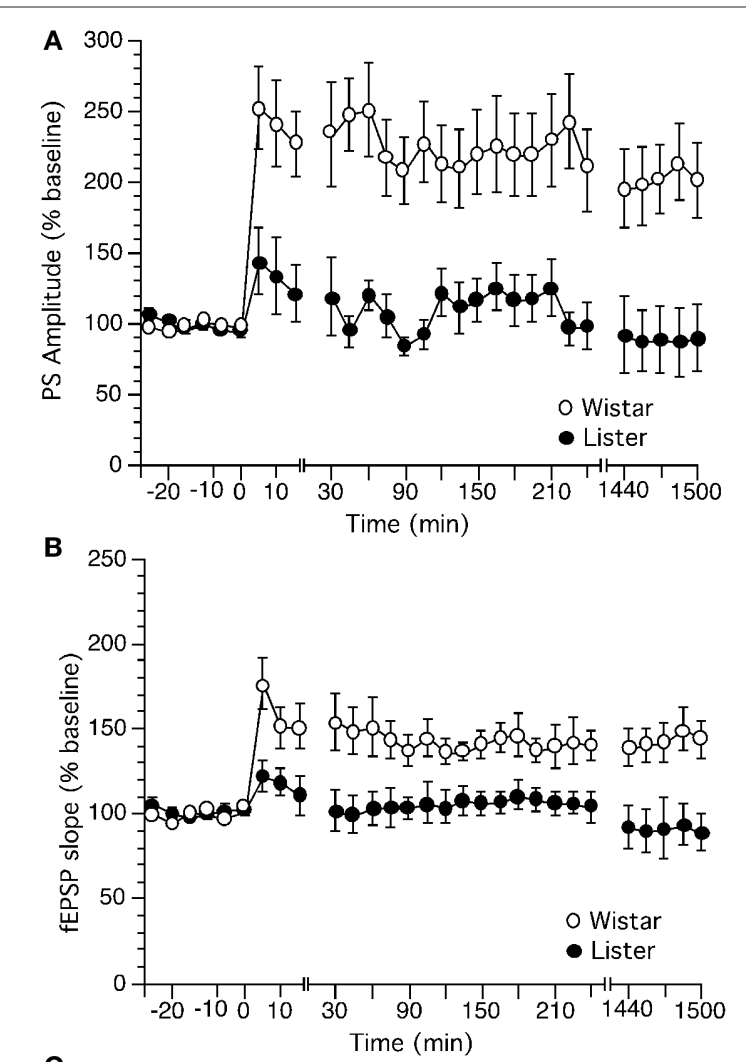

C
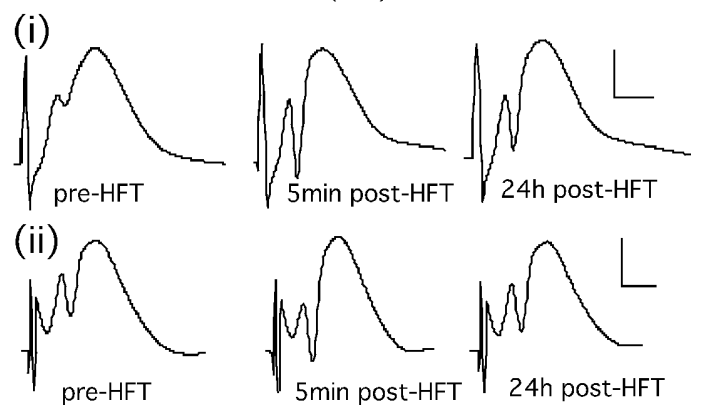

FIGURE 3 | High frequency stimulation induces long-term potentiation in the dentate gyrus of Wistar but not Hooded Lister rats. (A) High frequency stimulation (HFS, given at timepoint "O") at $200 \mathrm{~Hz}$ produces long-term potentiation which can be followed for over $24 \mathrm{~h}$ in Wistar rats. (B) HFS (given at timepoint " 0 ") at $200 \mathrm{~Hz}$ produces a transient potentiation which in HL rats which is not significantly different from test-pulse stimulated controls (shown in

Figure 2). (C) Original analog traces showing the field potentials evoked from the dentate gyrus pre HFT, 5 min, and $24 \mathrm{~h}$ following HFT in (i) Wistar or (ii) HL rats. Vertical scale-bar corresponds to $5 \mathrm{mV}$, horizontal scale-bar to $4 \mathrm{~ms}$.

Long-term potentiation in HL rats was not significantly different from baseline controls (each $n=11$ ). [ANOVA, for PS, within factor: $F(1,28)=0.381, p=0.5568$; between factor: $F(1,28)=1.509$, $p=0.0568$; for fEPSP, within factor: $F(1,28)=0.459, p=0.5234$; between factor: $F(1,28)=9.972, p=0.5108]$. The response to HFS in HL rats was furthermore significantly different from the response seen in Wistar rats. LTP in Wistar rats was significantly different from baseline controls [ANOVA, for PS, within factor: $F(1,28)=18.864, p=0.0015$; between factor: $F(1,28)=11.498, p=0.0001]$. 


\section{LOW FREOUENCY STIMULATION INDUCES ROBUST LTD IN WISTAR BUT} NOT HOODED LISTER RATS

Low frequency stimulation (LFS) at $1 \mathrm{~Hz}$ (900 pulses) produced persistent LTD in the dentate gyrus Wistar $(n=14)$ and HL rats $(n=11$; Figure 4). HL rats expressed synaptic depression which was not significantly different from Wistar rats, although the LTD expressed by HL rats appeared to be more decremental
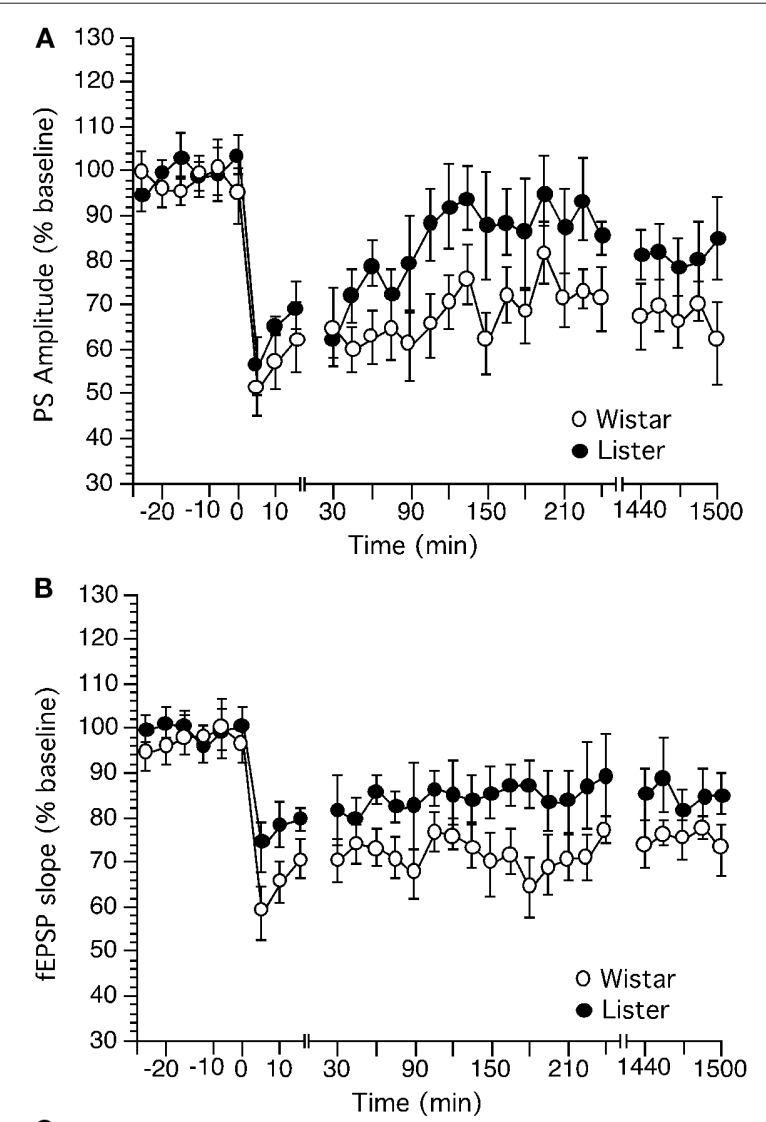

C
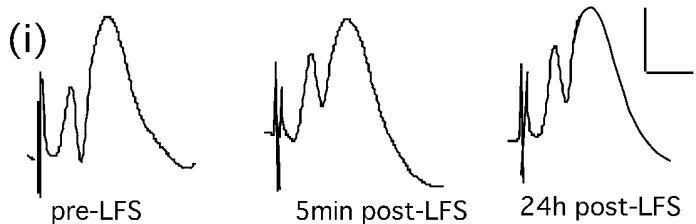

(ii)

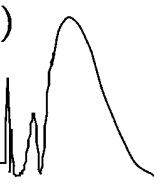

pre-LFS

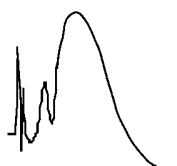

$5 \mathrm{~min}$ post-LFS

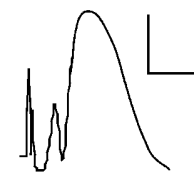

24h post-LFS

FIGURE 4 | Low frequency stimulation induces long-term depression in the dentate gyrus of Wistar rats that is more robust than LTD in Hooded Lister rats. (A) Low frequency stimulation (LFS, given at timepoint " 0 ") at $1 \mathrm{~Hz}$ (900 pulses) produces long-term depression which can be followed for over $24 \mathrm{~h}$ in Wistar rats. (B) LFS (given at timepoint "0") at $1 \mathrm{~Hz}$ (900 pulses) produces weaker depression in $\mathrm{HL}$ rats. (C) Original analog traces showing the field potentials evoked from the dentate gyrus pre LFS, 5 min, and $24 \mathrm{~h}$ following LFS in (i) Wistar or (ii) HL rats. Vertical scale-bar corresponds to $5 \mathrm{mV}$, horizontal scale-bar to $4 \mathrm{~ms}$.

than that seen in Wistars [Figure 4; ANOVA, for PS, within factor: $F(1,28)=1.66, p=0.2385$; between factor: $F(1,28)=0.609$, $p=0.9397$; for fEPSP, $F(1,28)=2.791, p=0.1459$; between factor: $F(1,28)=1.478, p=0.0693]$.

Long-term depression in Wistar rats was significantly different from baseline controls [ANOVA, for PS, within factor: $F(1,28)=101.301, p=0.0001$; between factor: $F(1,28)=4.532$, $p=0.0001$; for fEPSP, $F(1,28)=39.29, p=0.0008$; between factor: $F(1,28)=3.637, p=0.0001]$. Synaptic depression in HL rats was not significantly different from basally evoked responses (not shown), reflecting the greater variability of evoked responses in this strain after application of LFS. [ANOVA, for PS, within factor: $F(1,28)=0.296, p=0.601$; between factor: $F(1,28)=0.151$, $p=1.0$; for fEPSP, $F(1,28)=3.364, p=0.106$; between factor: $F(1,28)=0.577, p=0.9528]$.

\section{STRAIN-DEPENDENT DIFFERENCES IN SPATIAL LEARNING PERFORMANCE ARE EVIDENT IN WISTAR AND HOODED LISTAR RATS}

In the 8-arm radial maze, working memory performance was worse on days $1,2-4$, and $5-7$ in the Wistar rats $(n=10)$ compared to HL rats $(n=9$; Figure $5 \mathrm{~A}$; $t$-test $p<0.05)$ and in general, a significantly poorer working memory performance was seen in the Wistar strain on these days [ANOVA: $F(1,3)=12.558, p<0.01]$. No significant difference in working memory performance was evident on the final trial days (8-10) however (Figure 5A).

Wistar rats made more reference memory errors than HL rats on day $1(t$-test $p<0.01)$ and showed a significantly different reference memory performance across trial days [ANOVA: $F(1,3)=7.062$, $p<0.05]$. Interestingly, by days 5-7 performance was equal in both strains but by days 8-10 Wistar reference memory performance was significantly better than in the HL rat strain ( $t$-test, $p<0.05$; Figure 5B).

Double memory errors were higher in Wistar rats on day 1 $(t$-test, $p<0.05)$ but not significantly different from HL rats on the remaining trial days [Figure 5C; Mann-Whitney $\mathrm{U}\left(n_{1}=9\right.$, $\left.\left.n_{2}=10\right)=19\right]$. An overall difference in performance was seen with regard to double memory errors however [ANOVA: $F(1,3)=7.002, p<0.05]$.

Wistar rats groomed significantly more than HL rats throughout the entire trial period [ANOVA: $F(1,3)=22.341, p<0.001$, Figure $6 \mathrm{~A}$ ] and produced significantly more fecal boli [ANOVA: $F(1,3)=15.331, p<0.001$; Figure 6 C]. Wistar rats were significantly less mobile in the maze ( $p<0.001$, Figure 6B).

\section{DISCUSSION}

This study demonstrates that Wistar and HL rats exhibit strikingly different levels of synaptic plasticity in response to the same stimulation protocols: HL rats expressed more transient, and smaller, plasticity than Wistar rats that, in contrast, exhibited large, robust, and persistent $(>24 \mathrm{~h}$ ) plasticity. This was not paralleled by a clear correlation in behavioral performance. Spatial working memory was better in HL rats in the first days of training, but was not different toward the end of the study. On the other hand, a significant difference in reference memory was seen that was confined to the final days of training. Here, Wistar rats performed better in the reference memory task than the HL rats. 

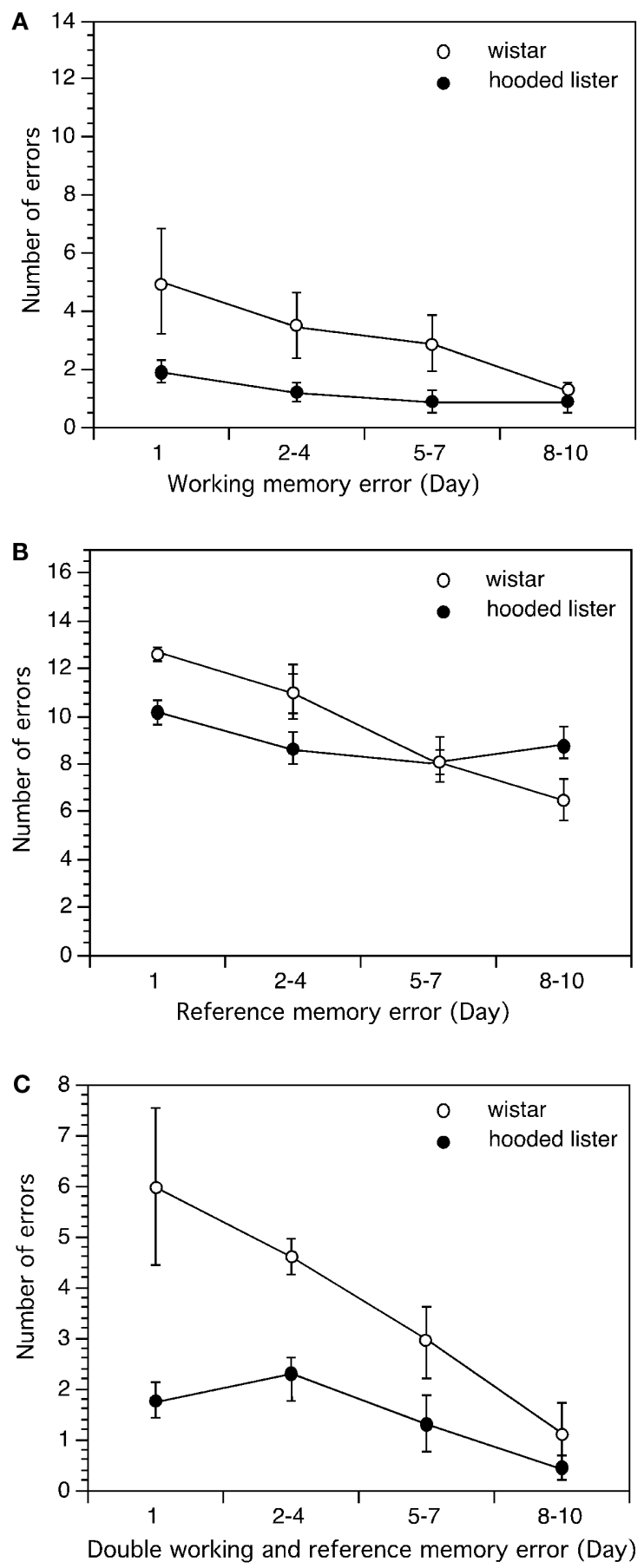

FIGURE 5 | Comparison of learning performance in Wistar and Hooded Lister rats in the eight-arm radial maze. Spatial learning was not different when Wistar and HL rats were compared with regard to working memory (A). However, Wistar rats showed significantly better reference memory (B) and double memory performance (C) compared to HL rats. Graphs show the number of errors ( $y$-axis) across the trial blocks of the study ( $x$-axis).

The differences in synaptic plasticity seen in the study may correspond to a difference in the ability of the animals to engage in presynaptic glutamate release: we observed a significant difference in paired-pulse responses in the 40 - to 120 -ms interpulse interval
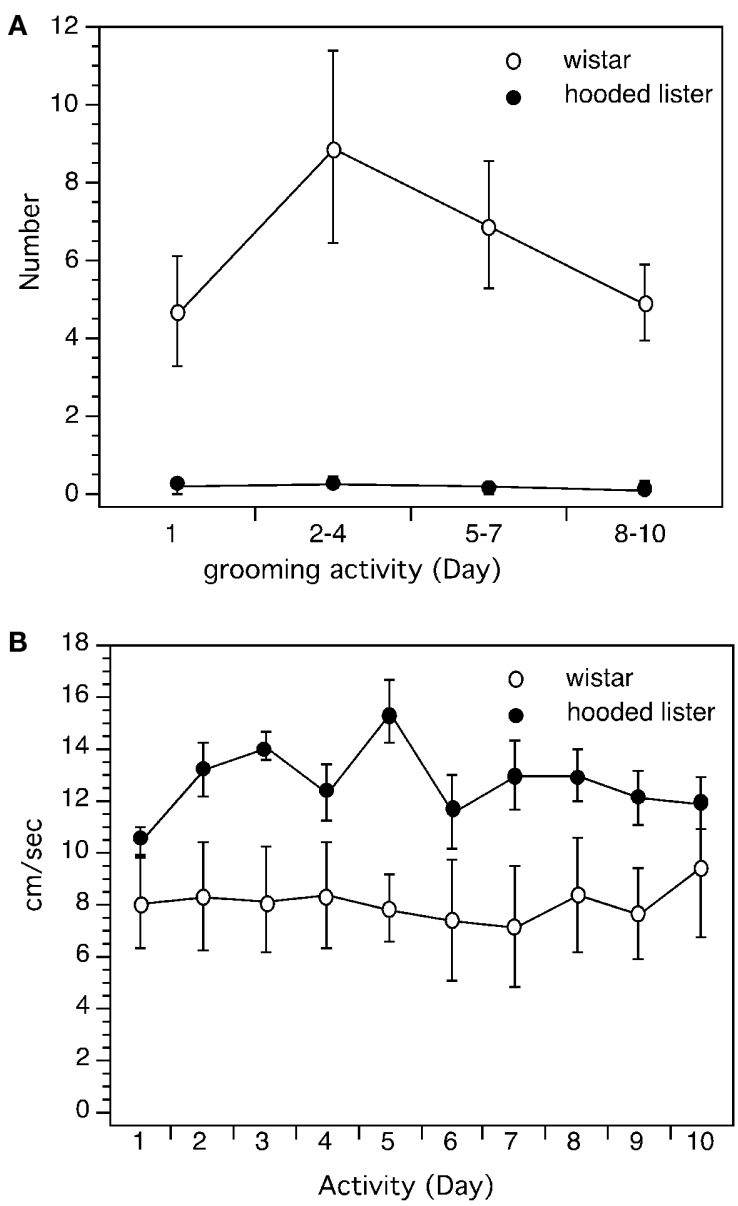

C

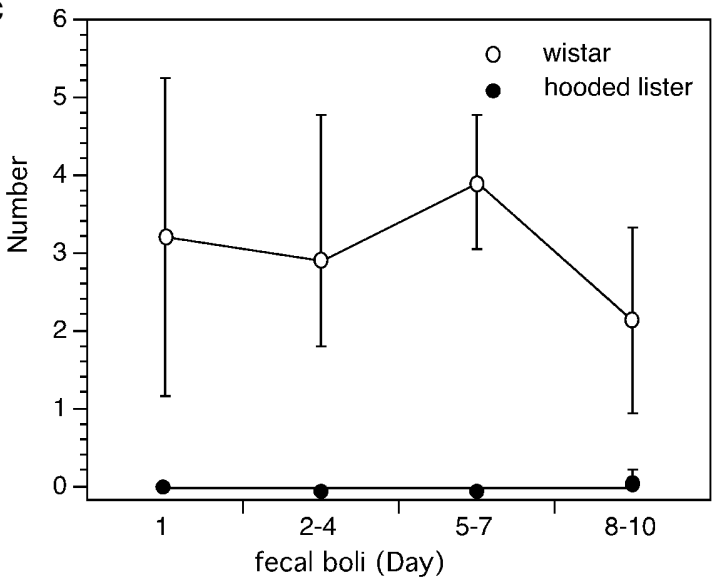

FIGURE 6 | Comparison of behavioral measures in Wistar and Hooded Lister rats. Analysis of behavioral performance revealed that Wistar rats were less active in the maze than $\mathrm{HL}$ rats. Wistar rats undertook more grooming activity (A), less locomotory activity (B), and produced more fecal boli (C) than HL rats.

range. Facilitation obtained with paired-pulse intervals of 40-120 ms reflects an increase of NMDA-mediated responses to further glutamate release from the presynapse (McNaughton, 1982; Albertson and Joy, 1987) but also may be influenced by activation of GABAb 
receptors (Kahle and Cotman, 1993). This suggests that HL may release less presynaptic glutamate compared to Wistar rats. This might in turn explain the higher thresholds for synaptic plasticity seen in the HL strain and might also partly explain the strain-dependent differences in spatial learning seen in the present study (Richter-Levin et al., 1995). These differences cannot be explained by variabilities in the sensitivity of the input/output relationships of the animal strains, as these were not significantly different between strains. In line with the possibility that differences in presynaptic glutamate release may underline the differences in synaptic plasticity seen in the present study, stable LTD is expressed in the CA1 region of HL rats in response to the same LFS, if (presynaptic) group III (adenylyl cyclase coupled) metabotropic glutamate receptors (mGluR) are activated with an agonist (Manahan-Vaughan, 2000b). Furthermore, Wistar and HL strains exhibit a different regulation of synaptic plasticity and spatial learning by mGluR5 (Manahan-Vaughan and Braunewell, 2005), suggesting that differences in neurotransmitter function or expression may underlie the strain-dependent plasticity differences observed. Similarly, in mice, strain-dependent differences in plasticity-regulated proteins have been reported, which may also contribute to the effects seen here (Pollak et al., 2005, 2010).

Differences in the expression of hippocampal synaptic plasticity in Wistar and HL rats have been previously described for the CA1 region, where a strain-dependent frequency-dependency of synaptic plasticity is also evident (Manahan-Vaughan and Braunewell, 1999, 2005; Manahan-Vaughan, 2000a,b). Whereas Wistar rats express robust LTP in response to $100 \mathrm{~Hz}$ stimulation of this hippocampal subregion, HL rats exhibit an LTP which, in the induction phase, is of lower magnitude but gradually increases to Wistar levels after about $2 \mathrm{~h}$. Wistar rats respond to LFS $(1-3 \mathrm{~Hz})$ with robust LTD in the CA1 region, whereas HL rats express only short-term depression (STD). In the present study striking differences were also observed in the plasticity expressed by these strains in the dentate gyrus. Here, we observed that HFS $(200 \mathrm{~Hz})$ elicited robust LTP that lasted for over $24 \mathrm{~h}$ in Wistar rats. In HL rats the same HFS protocol elicited a very small short-term potentiation that endured for ca. $30 \mathrm{~min}$. Similar to the CA1 region, LFS elicited persistent LTD in Wistar and a less robust synaptic depression in HL rats. These differences in strain-dependent synaptic plasticity in the CA1 region and dentate gyrus may reflect the degree of specialization of the hippocampal regions in the control of behavior. Indeed, multiple studies support the likelihood of distinct roles for the hippocampal subregions in spatial information processing. The dentate gyrus is believed to play an important role in pattern separation (Leutgeb et al., 2007; Hunsaker and Kesner, 2008; Myers and Scharfman, 2009) whereas the CA1 region functions in the temporal integration of information (Fortin et al., 2002; Hunsaker and Kesner, 2006, 2008; Hoge and Kesner, 2007; Vago et al., 2007). In the past, we have shown that LTP and LTD are associated with different precise components of a spatial representation (ManahanVaughan and Braunewell, 1999; Kemp and Manahan-Vaughan, 2004, 2007) and that hippocampal LTD in the CA1 region and dentate gyrus, for example, is facilitated by very different aspects of a spatial context (Kemp and Manahan-Vaughan, 2008). Another factor to consider is whether short-term and long-memory are contiguous. Recent evidence suggests a dissociation between these learning phenomena (Sanderson et al., 2009), and support that deficits in LTP are associated with deficits in spatial reference memory (Rust et al., 2010, but see also Niewoehner et al., 2007). This finding is in line with our observation that HL rats exhibit impaired synaptic potentiation and reference memory in comparison to Wistar rats. Nonetheless, one cannot simply presume that short-term memory is enabled by STP and that long-term memory is enabled by LTP. Studies in transgenic mice have shown that enhanced LTP can be accompanied by marked deficits in spatial learning (Shimshek et al., 2006): but here, one needs to consider that saturated LTP may also impair learning (Moser et al., 1998). Thus, the strain-dependent differences seen in these hippocampal subregions may reflect on the one hand, subregional specializations in information processing and, on the other hand, related strain-dependent differences in the ability to process spatial learning through synaptic plasticity.

The behavioral tool we chose to evaluate this latter postulate, was possibly too generalized and perhaps even too easy to enable a precise discrimination of spatial memory components. In other studies, very clear differences in spatial learning ability across rats strains have been reported - although Wistar and HL rats were not compared (Gleason et al., 1999; Albasser et al., 2010). In the present spatial working memory task, the animals simply had to remember from which arm they had removed a food pellet; a task that should be hippocampus-dependent (Nadel and MacDonald, 1980). Thus, they engaged in short-term acquisition of trial-specific information (Baddeley, 1981, Cowan et al., 2008). In the reference memory task, they needed to remember which arms were never "baited"; this comprised a long-term memory task. The relatively flat learning curve of the HL rats in the working memory task reflects a ready ability to grasp this task. This is something we also observed in a previous study (Manahan-Vaughan and Braunewell, 2005), although here, the variability across responses between HL and Wistar rats was such that no significant difference in working memory was evident in the first week of training (in contrast to the present study). This is likely explained by the fact that the previous and current studies were not conducted in the same environment, i.e., the location, spatial cues, and conditions of the environments were not identical. This can have a marked effect on the outcome of hippocampal information processing (Colgin et al., 2008). Although working memory reflects the ability of an animal to hold information "online," it is also influenced by the performance of an animal within a given trial (Olton and Papas, 1979). The steeper learning curve of the Wistar rats may thus reflect their lower activity levels and higher relative anxiety. Be that as it may, the $\mathrm{HL}$ rats performed better in the working memory task across the study, although the Wistar rats reached the HL performance level in the last 3 days. This suggests that habituation to the task resulted in an improvement in working memory performance in the Wistar rats.

In terms of reference memory performance, the Wistar rats also performed better than the HL strain in the last 3 days of the study. This is in line with our findings in a previous study (ManahanVaughan and Braunewell, 2005). In the earlier days of the study, the performance of the HL rats were initially better (days 1-4), and subsequently equivalent (days 5-7). Nonetheless, by the final days of the study Wistar rats exhibited significantly fewer reference memory errors. The picture is different when "double" working and reference memory errors were assessed. This has a reference memory component because the animals should remember that the arm is not baited, but also has a working memory component because the 
animal have already erroneously entered an unbaited arm (Mizumori et al., 1987; Crusio et al., 1993). Here, the HL rats were better at the task - presumably because of the working memory component.

\section{CONCLUSION}

Taken together with our behavioral data, the differences in synaptic plasticity seen may reflect differences in the cognitive abilities of these rat strains. In the eight-arm radial maze, Wistar rats exhibited an overall better reference memory performance compared to HL rats. Working memory in the strains was initially better in the HL rats, but by the final day of training, Wistar rats performed equally well as HL rats. It is tempting to speculate that the differences in spatial memory seen in these rats may derive from differences in synaptic plasticity occurring both in the CA1 region (Manahan-Vaughan

\section{REFERENCES}

Abraham, W. C. (2003). How long will log-term potentiation last? Philos. Trans. R. Soc. Lond., B, Biol. Sci. 358, 735-744.

Albasser, M. M., Chapman, R. J., Amin, E., Iordanova, M. D., Vann, S. D., and Aggleton, J. P. (2010). New behavioral protocols to extend our knowledge of rodent object recognition memory. Learn. Mem. 17, 407-419.

Albertson, T. E., and Joy, R. M. (1987). Increased inhibition in dentate gyrus granule cells following exposure to GABA-uptake blockers. Brain Res. 435, 283-292.

Baddeley,A. (1981). The concept of working memory: a view of its current state and probable future development. Cognition 10, 17-23.

Bear M, F., and Abraham, W. C. (1996). Long-term depression in the hippocampus. Annu. Rev. Neurosci. 19, 437-462.

Bliss, T. V., and Collingridge, G. L. (1993). A synaptic model of memory: longterm potentiation in the hippocampus. Nature 361, 31-39.

Colgin, L. L., Moser, E. I., and Moser, M. B. (2008). Understanding memory through hippocampal remapping. Trends Neurosci. 31, 469-477.

Cowan, N., Morey, C. C., Chen, Z., Gilchrist, A. L., and Saults, J. S. (2008). "Theory and measurement of working memory capacity limits," in The Psychology of Learning and Motivation, Vol. 49, ed. B. H. Ross (Amsterdam: Elsevier), 49-104.

Crusio, W. E., Schwegler, H., and Brust, I. (1993). Covariations between hippocampal mossy fibres and working and reference memory in spatial and non-spatial radial maze tasks in mice. Eur. J. Neurosci. 5, 1413-1420.

Eckert, M. J., Bilkey, D. K., and Abraham, W.C. (2010). Altered plasticity in hippocampal CA1, but not dentate gyrus, following long-term environmental enrichment. J. Neurophysiol. 103, 3320-3329.

Etkin, A., Alarcón, J. M., Weisberg, S. P., Touzani, K., Huang, Y. Y., Nordheim, A., and Kandel, E. R. (2006). A role in learning for SRF: deletion in the adult forebrain disrupts LTD and the formation of an immediate memory of a novel context. Neuron 50, 127-143.

Fortin, N.J.,Agster, K.L., and Eichenbaum, H. B. (2002). Critical role of the hippocampus in memory for sequences of events. Nat. Neurosci. 5, 458-462.

Frey, U., Frey, S., Schollmeier, F., and Krug, M. (1996). Influence of actinomycin D, a RNA synthesis inhibitor, on longterm potentiation in rat hippocampal neurons in vivo and in vitro. J. Physiol. 490, 703-711.

Frey, U., Krug, M., Reymann, K. G., and Matthies, H. (1988). Anisomycin, an inhibitor of protein synthesis, blocks late phases of LTP phenomena in the hippocampal CAl region in vitro. Brain Res. 452, 57-65.

Ge, Y., Dong, Z., Bagot, R. C., Howland, J. G., Phillips, A. G., Wong, T. P., and Wang, Y. T. (2010). Hippocampal long-term depression is required for the consolidation of spatial memory. Proc. Natl. Acad. Sci. U.S.A. 107, 16697-16702.

Gleason, T. C., Dreiling, J. L., and Crawley, J. N. (1999). Rat strain differences in response to galanin on the Morris water task. Neuropeptides 33, 265-270.

Hoge, J., and Kesner, R. P. (2007). Role of $\mathrm{CA} 3$ and $\mathrm{CA} 1$ subregions of the dorsal hippocampus on temporal processing of objects. Neurobiol. Learn Mem. 88, 225-231.

Hunsaker, M. R., and Kesner, R. P. (2006). The role of CA 3 and CA1 in the acquisition of an object-trace-place pairedassociate task. Behav. Neurosci. 120, $1252-1256$.

Hunsaker, M. R., and Kesner, R. P. (2008). Evaluating the differential roles of the

and Braunewell, 1999; Manahan-Vaughan, 2000a), and in the dentate gyrus, as reported here. Our behavioral data are in line with previous reports that describe better reference memory performance in Wistar compared to HL rats, that HL rats tend to master a working memory task faster than Wistar rats (Manahan-Vaughan and Braunewell, 2005), and that LTP and LTD in the CA1 region and dentate gyrus are correlated to spatial memory performance (Nakao et al., 2001, 2002; Ge et al., 2010). Whether the differences in synaptic plasticity are directly correlated to strain-dependent cognitive differences remains to be determined.

\section{ACKNOWLEDGMENT}

Grant sponsor: Deutsche Forschungsgemeinschaft (Ma 1843; SFB 779 TP5 to Herbert Schwegler).

dorsal dentate gyrus, dorsal CA3, and dorsal CA1 during a temporal ordering for spatial locations task. Hippocampus $18,955-964$.

Kahle, J. S., and Cotman, C. W. (1993). Adenosine, L-AP4, and baclofen modulation of paired-pulse potentiation in the dentate gyrus: interstimulus interval-dependent pharmacology. Exp. Brain Res. 94, 97-104.

Kemp, A., and Manahan-Vaughan, D. (2004). Hippocampal long-term depression and long-term potentiation encode different aspects of novelty acquisition. Proc. Natl. Acad. Sci. U.S.A. 101, 8192-8197.

Kemp, A., and Manahan-Vaughan, D. (2007). Hippocampal long-term depression: master or minion of declarative memory processes. Trends Neurosci. 30, 111-118.

Kemp, A., and Manahan-Vaughan, D. (2008). The hippocampal CA1 region and dentate gyrus differentiate between environmental and spatial feature encoding through long-term depression. Cereb. Cortex 18, 968-977.

Kulla, A., and Manahan-Vaughan, D. (2000). Depotentiation in the dentate gyrus of freely moving rats is modulated by D1/D5 dopamine receptors. Cereb. Cortex 10, 614-620.

Leutgeb, J. K., Leutgeb, S., Moser, M. B., and Moser, E. I. (2007). Pattern separation in the dentate gyrus and CA3 of the hippocampus. Science 315, 961-966.

Manahan-Vaughan, D. (2000a). Longterm depression in freely moving rats is dependent upon strain variation, induction protocol and behavioral state. Cereb. Cortex 10, 482-487.

Manahan-Vaughan, D. (2000b). Group III metabotropic glutamate receptors modulate long-term depression in the hippocampal CA1 region of two rat strains in vivo. Neuropharmacology39, 1952-1958.

Manahan-Vaughan, D., and Braunewell, K. H. (1999). Novelty acquisition is asso- ciated with induction of hippocampal long-term depression. Proc. Natl. Acad. Sci. U.S.A. 96, 8739-8744.

Manahan-Vaughan, D., and Braunewell, K. H. (2005). The metabotropic glutamate receptor, mGluR5, is a key determinant of good and bad spatial learning performance and hippocampal synaptic plasticity. Cereb. Cortex 15, 1703-1713.

Manahan-Vaughan, D., Braunewell, K. H., and Reymann, K. G. (1998). Subtype-specific involvement of metabotropic glutamate receptors in two forms of long-term potentiation in the dentate gyrus of freely moving rats. Neuroscience 86, 709-721.

Manahan-Vaughan, D., Kulla, A., and Frey, J. U. (2000). Requirement of translation but not transcription for the maintenance of long-term depression in the CA1 region of freely moving rats. J. Neurosci. 20, 8572-8576.

McNaughton, B. L. (1982). Long-term synaptic enhancement and shortterm potentiation in rat fascia dentata act through different mechanisms. J. Physiol. (Lond.) 324, 249-262.

Mizumori, S. J. Y., Channon, V., Rosenzweig, M. R., and Bennett, E. L. (1987).Short- and long-term components of working memory in the rat. Behav. Neurosci. 101, 782-789.

Moser, E. I., Krobert, K. A., Moser, M. B., and Morris, R. G. (1998). Impaired spatial learning after saturation of long-term potentiation. Science 281, 2038-2042.

Myers, C. E., and Scharfman, H.E. (2009). A role for hilar cells in pattern separation in the dentate gyrus: a computational approach. Hippocampus 19 , 321-337.

Nadel, L., and MacDonald, L. (1980). Hippocampus: cognitive map or working memory? Behav. Neural Biol. 29, 405-409.

Nakao, K., Ikegaya, Y., Yamada, M. K., Nishiyama, N., and Matsuki, N. 
(2001). Spatial performance correlates with long-term potentiation of the dentate gyrus but not of the CA1 region in rats with fimbria-fornix lesions. Neurosci. Lett. 307, 159-162.

Nakao, K., Ikegaya, Y., Yamada, M. K., Nishiyama, N., and Matsuki, N. (2002). Hippocampal long-term depression as an index of spatial working memory. Eur. J. Neurosci. 16, 970-974.

Nguyen, P. V., Abel, T., and Kandel, E. R. (1994). Requirement of a critical period of transcription for induction of a late phase of LTP. Science 265, 1104-1107.

Niewoehner, B., Single, F. N., Hvalby, $\varnothing$., Jensen, V., Meyer zum Alten Borgloh, S., Seeburg, P. H., Rawlins, J. N., Sprengel, R., and Bannerman, D. M. (2007). Impaired spatial working memory but spared spatial reference memory following functional loss of NMDA receptors in the dentate gyrus. Eur. J. Neurosci. 25, 837-846.

Olton, D. S., and Papas, B. C. (1979). Spatial memory and hippocampal function. Neuropsychologia 17, 669-682.

Ossenkopp, K.-P., Macrae, L. K., and Teskey, C. G. (1987). Automated multivariate measurement of spontaneous motor activity in mice: time course and reliabilities of the behavioral measures. Pharmacol. Biochem. Behav. 27, 565-568.

Otani, S., Marshall, C. J., Tate, W. P., Goddard, G. V., and Abraham, W. C. (1989). Maintenance of long-term potentiation in rat dentate gyrus requires protein synthesis but not messenger RNA synthesis immediately post-tetanization. Neuroscience 28, 519-526.

Pollak, D. D., Bae, N., Mostafa, G., and Hoeger, H. (2010). Strain-dependent expression of metabolic proteins in the mouse hippocampus. Amino Acids.39, 1451-1462.

Pollak, D. D., Scharl, T., Leisch, F., Herkner, K., Villar, S. R., Hoeger, H., and Lubec, G. (2005). Strain-dependent regulation of plasticity-related proteins in the mouse hippocampus. Behav. Brain Res. 165, 240-246.

Prior, H., Schwegler, H., and Dücker, G. (1997). Dissociation of spatial reference memory, spatial working memory, and hippocampal mossy fiber distribution in two rat strains differing in emotionality. Behav. Brain Res. 87, 183-194.

Richter-Levin, G., Canevari, L., and Bliss, T. V. (1995). Long-term potentiation and glutamate release in the dentate gyrus: links to spatial learning. Behav. Brain Res. 66, 37-40.

Rust, M. B., Gurniak, C. B., Renner, M., Vara, H., Morando, L., Görlich, A., Sassoè-Pognetto, M., Al Banchaabouchi, M., Giustetto, M. Triller, A., Choquet, D., and Witke, W. (2010). Learning, AMPA receptor mobility and synaptic plasticity depend on n-cofilin mediated actin dynamics. EMBO J. 29, 1889-1902.

Sanderson, D. J., Good, M. A., Skelton, K. Sprengel, R., Seeburg, P. H., Rawlins, J. N., and Bannerman, D. M. (2009). Enhanced long-term and impaired short-term spatial memory in GluA1 AMPA receptor subunit knockout mice: evidence for a dual-process memory model. Learn. Mem. 16, 379-386.

Shimshek, D. R., Jensen, V., Celikel, T., Geng, Y., Schupp, B., Bus, T. Mack, V., Marx, V., Hvalby, O. Seeburg, P. H., and Sprengel, R. (2006). Forebrain-specific glutamate receptor $\mathrm{B}$ deletion impairs spatial memory but not hippocampal field long-term potentiation. J. Neurosci. 26, 8428-8440.

Straube, T., Korz, V., and Frey, J. U. (2003) Bidirectional modulation of longterm potentiation by novelty-explo- ration in rat dentate gyrus. Neurosci. Lett. 2344, 5-8.

Vago, D. R., Bevan, A., and Kesner, R. P. (2007). The role of the direct perforant path input to the CAl subregion of the dorsal hippocampus in memory retention and retrieval. Hippocampus 17, 977-987.

Conflict of Interest Statement: The authors declare that the research was conducted in the absence of any commercial or financial relationships that could be construed as a potential conflict of interest.

Received: 17 September 2010; accepted: 11 Februay 2011; published online: 07 March 2011.

Citation: Manahan-Vaughan D and Schwegler H (2011) Strain-dependent variations in spatial learning and in hippocampal synaptic plasticity in the dentate gyrus of freely behaving rats. Front. Behav. Neurosci. 5:7. doi: 10.3389/fnbeh.2011.00007

Copyright $(2011$ Manahan-Vaughan and Schwegler. This is an open-access article subject to an exclusive license agreement between the authors and Frontiers Media $S A$, which permits unrestricted use, distribution, and reproduction in any medium, provided the original authors and source are credited. 